Palavras chave:

Histórico:

Recebido 12/04/2012

Aceito 13/12/2013

Keywords:

LAI

VAI

Hemispherical photos

LAI-2000

Eucalyptus

Correspondência: cantinho@dsr.inpe.br
Roberta Zecchini Cantinho', Flávio Jorge Ponzoni², Sandra Benfica dos Santos ${ }^{2}$

\section{ESTIMATIVAS DE ÍNDICE DE ÁREA FOLIAR DE PLANTIOS DE Eucalyptus spp. SEGUNDO TRÊS ALTERNATIVAS METODOLÓGICAS}

RESUMO: Foram realizadas estimativas de Índice de Área Foliar (IAF) e de Índice de Área de Vegetação (IAV) em 36 parcelas posicionadas em plantios de Eucalyptus sp. localizados nos municípios de Aracruz e São Mateus (ES), de propriedade da empresa FIBRIA S/A, mediante a aplicação de três diferentes técnicas: método destrutivo, processamento de fotografias hemisféricas tomadas no interior dos dosséis e utilização do equipamento LAl2000 (LICOR). As coletas de dados em campo foram realizadas em duas épocas do ano, procurando explorar diferenças fenológicas atuantes principalmente sobre os valores de IAF e de IAV. As estimativas de IAF efetuadas a partir do método destrutivo, em termos absolutos, foram sempre superiores àquelas do IAV efetuadas a partir dos métodos indiretos. No período chuvoso, as relações entre as estimativas de IAF e de IAV, para ambos os métodos indiretos, foram mais fortes e consistentes do que aquelas verificadas no período mais seco do ano. As relações entre as estimativas de IAV efetuadas pelos dois métodos indiretos (fotografias e LAl-2000) foram consistentes e com grau de aderência mais elevado do que aquelas verificadas entre o IAF e IAV.

\section{LEAF AREA INDEX ESTIMATION OF EUCALYPTUS PLANTATION USING THREE ALTERNATIVE METHODOLOGIES}

ABSTRACT: Leaf Area Index (LAl) and Vegetation Area Index (VAl) estimations have been performed in 36 sample points located in Eucalyptus sp. canopies situated in Aracruz and São Mateus (ES) towns and from FIBRIA S/A company. Three different techniques have been applied: destructive method, hemispherical photos taken inside the canopies and LAI-2000 (LICOR). The field data collections were carried out in two seasons in order to detect phenological changes that should affect both LAI and VAI values. LAI values estimated by destructive method were higher than VAl values estimated by the indirect methods in absolute terms. During the rainy season the relationships between LAI and $\mathrm{VAl}$, for the two indirect methods, were stronger and more consistent than those verified for the dry season. The relationships between VAl (indirect methods) were consistent than those calculated between LAI and VAI. 


\section{INTRODUÇÃO}

O aumento da demanda de plantações florestais e a busca pelo incremento da produtividade levaram à necessidade do conhecimento da ecofisiologia das espécies e da limitação dos recursos naturais no seu crescimento (STAPE, 2004). A ecofisiologia aborda os processos e respostas vitais das plantas em função das mudanças nas condições ambientais (LARCHER, 2006). Fatores climáticos como a radiação solar, a disponibilidade hídrica e a temperatura do ar influenciam direta ou indiretamente os processos fisiológicos e bioquímicos, como a fotossíntese, a transpiração, a respiração e a divisão celular (MAESTRI, 2003; O'BRIEN, 1995; SOUZA et al., 2006). Segundo Lowman e Nadkarni (1995), a grande maioria desses processos ocorre no dossel florestal e está envolvida com o crescimento e com o desenvolvimento das plantas. Dessa forma, a caracterização estrutural da copa das árvores e o modo como a radiação solar é interceptada e sintetizada pelo dossel é de grande importância para estudos ecofisiológicos, pois atuam diretamente sobre as funções que controlam a produtividade (CAMPBELL; NORMAN, 1989).

O desenvolvimento da copa e sua atividade fisiológica podem ser avaliados pela medição da quantidade de radiação solar interceptada pelas folhas do dossel e sua eficiência de uso na forma de produção de biomassa florestal (CHAVES et al., 2007; ROSS, 198I). O Índice de Área Foliar (IAF) é uma variável biofísica que apresenta estreita relação com a produtividade de uma comunidade vegetal, pois representa a capacidade da planta em explorar o espaço ambiental disponível (GOWER et al., 1999; LANG; MCMURTRIE, 1992; XAVIER; VETTORAZZI, 2003), sendo inclusive aproveitado como parâmetro de entrada em modelos relacionados à evapotranspiração, à simulação dos ciclos de carbono e de água e à produtividade florestal (RODRIGUEZ et al., 2009; STAPE et al., 2004). De acordo com Bréda (2003), o IAF pode ser considerado um elemento-chave na compreensão da dinâmica da vegetação em ecossistemas terrestres.

Geralmente, as metodologias utilizadas para a determinação desse parâmetro são indiretas, em razão das suas vantagens operacionais em relação ao método direto que, apesar de mais preciso, é mais trabalhoso e custoso (CHAVES et al., 2007; GOWER et al., 1999). No entanto, Jonckheere et al. (2004) acrescentam que o método destrutivo é importante para a calibração de técnicas indiretas e, em razão da elevada variação de área foliar entre plantas, o método demanda a amostragem de grande número de indivíduos em diferentes idades. Experimentos comprovaram a proximidade de resultados obtidos por meio de métodos diretos e indiretos (MACFARLANE et al., 2007; ZHANG et al., 2005).

Os instrumentos e técnicas desenvolvidos para a obtenção indireta do IAF são rápidos e fáceis na coleta de dados (CHAVES et al., 2007) e, conforme Jonckheere et al. (2004), esses analisadores de dossel hemisféricos, como o LAl-2000 e a câmera fotográfica dotada de lentes hemisféricas, obtêm o parâmetro por comparação diferencial entre a radiação abaixo e acima do dossel com base na análise do gap fraction (abertura do dossel). Como esse método não distingue os tecidos fotossinteticamente ativos de outros componentes do dossel, como galhos, ramos e troncos, adota-se a expressão Índice de Área de Vegetação (IAV) para referirse à estimativa obtida por essas metodologias (BRÉDA, 2003; JONCKHEERE et al., 2004).

Neste trabalho, objetivou-se avaliar três diferentes metodologias para a estimativa de IAF de dosséis florestais. Para isso, IAF e IAV de plantios de Eucalyptus spp. (eucalipto) pertencentes à empresa FIBRIA S/A foram estimados a partir da adoção de uma técnica destrutiva e de dois métodos indiretos - utilização do equipamento LAI-2000 e tomada de fotografias hemisféricas - em duas estações climáticas distintas do ano. Os diferentes métodos foram comparados entre si e validados com os dados estimados a partir do método direto.

\section{MATERIAL E MÉTODOS}

\section{Área de estudo}

O trabalho foi realizado em plantios de eucalipto pertencentes à Empresa FIBRIA S/A localizados nas Unidades Florestais de Aracruz e de São Mateus, que se encontram no Estado do Espírito Santo (Figura I).

O clima da região é classificado por Köppen como Aw, com temperatura e precipitação média anual de $23^{\circ}$ e $1.400 \mathrm{~mm}$ ao ano, respectivamente (OMETTO, 198I). Os dados obtidos das estações instaladas pela própria empresa indicam que o volume do regime de chuvas da região de Aracruz é um pouco maior do que a de São Mateus (Figura 2). As setas no gráfico apontam as épocas de coleta de dados em campo.

Os plantios com espaçamento $3 \times 3 \mathrm{~m}$ e de idades, variando entre 19 e 57 meses, encontram-se em área plana e, de acordo com o levantamento de reconhecimento dos solos pela empresa, predominam 


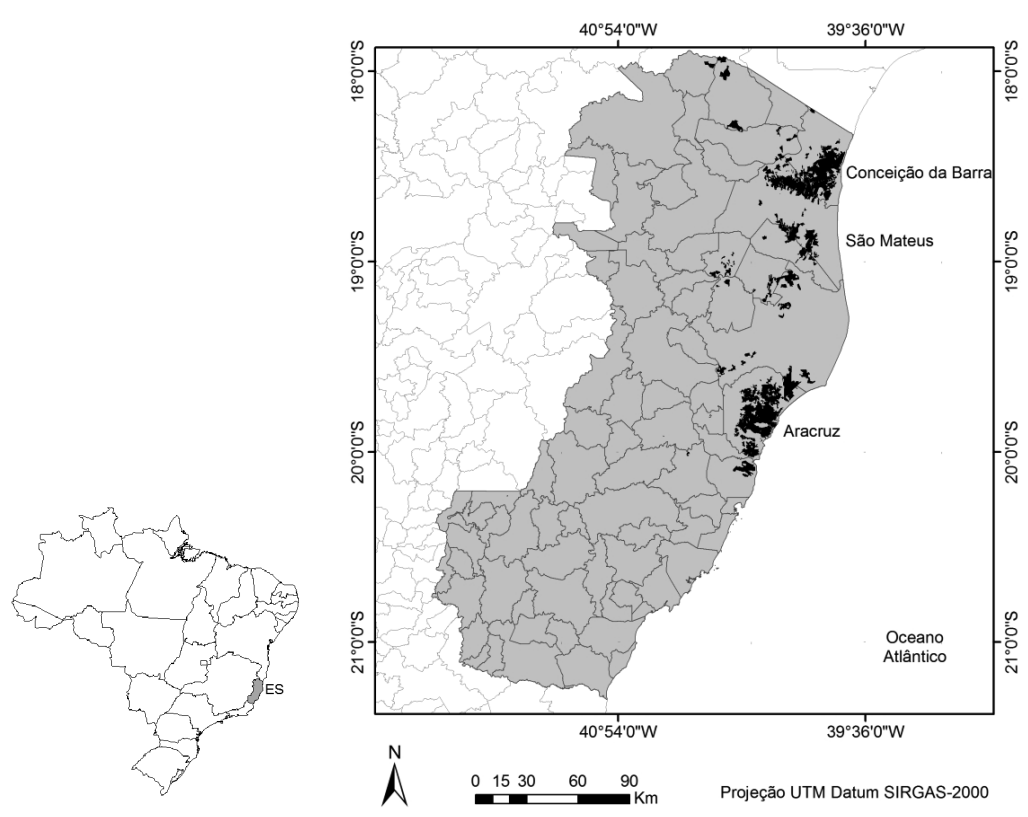

FIGURA I Localização geográfica dos talhões florestais das Unidades de Aracruz e São Mateus no Estado do Espírito Santo.

FIGURE I Geographical location of forest stands of the Units of Aracruz and São Mateus counties in the State of Espírito Santo.
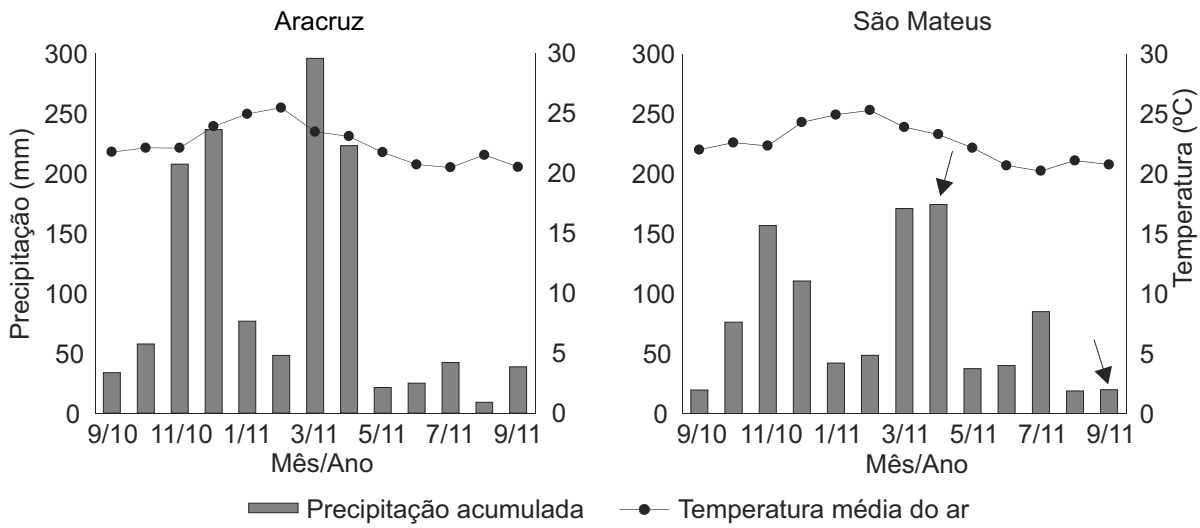

FIGURA 2 Precipitação e temperatura mensais médias das regiões de Aracruz e São Mateus. ( $\rightarrow$ : período de coleta de dados) FIGURE 2 Monthly average of precipitation and temperature of Aracruz and São Mateus regions. ( $\rightarrow$ : data collection period)

as classes Argissolo e Latossolo. Estendendo a classificação até o terceiro nível hierárquico (Grande Grupo) predominam associações de Argissolo-Amarelo distrófico, com textura média/argilosa, arenosa/argilosa, arenosa/média/argilosa e arenosa/média de relevo plano e de Latossolo-Amarelo coeso com textura média/argilosa e arenosa/média de relevo plano (EMPRESA BRASILEIRA DE PESQUISA AGROPECUÁRIA - EMBRAPA, 2006).

\section{Coleta de dados em campo}

Os trabalhos em campo foram realizados visando a adquirir dados que permitissem estimar IAF e IAV, segundo três alternativas metodológicas. As coletas foram efetuadas em diferentes períodos do ano, a fim de explorar diferenças fenológicas que implicassem em variações dos valores de IAF e de IAV nos talhões de eucalipto de diferentes idades. Tais variações vêm sendo relatadas em trabalhos como o de Le Maire et al. (20 I I). O primeiro trabalho de campo foi realizado entre 28 de março e 07 de abril de 201 I, quando foram definidas aleatoriamente 36 parcelas amostrais em talhões de eucalipto de diferentes idades, procurando, tanto quanto possível, explorar diversidade estrutural e arquitetônica. Cada parcela era circular com uma área de $400 \mathrm{~m}^{2}$, sendo seu centro definido também de forma aleatória, buscando posicioná-las o mais distante possível das bordas dos talhões. Dentro das parcelas, foram obtidas medidas de Diâmetro à Altura do Peito (DAP), Altura total $(\mathrm{H})$ e Altura da Copa $(\mathrm{Hc})$ de todas 
as árvores - aproximadamente 45 indivíduos. Ainda, tomando como referência o centro da parcela, foram selecionadas as 10 árvores mais próximas e, além do DAP, $\mathrm{H}$ e Hc, foram medidos os Raios da copa (tomado em dois eixos ortogonais entre $\mathrm{si}-\mathrm{Rcl}$ e Rc2).

Nas proximidades da parcela - fora de sua área de abrangência para não influenciar as futuras medidas dentro da parcela, mas ainda dentro do talhão - uma árvore foi selecionada e abatida para o cálculo do IAF. A seleção do indivíduo a ser abatido foi feita com base na média do DAP das 45 árvores medidas dentro da parcela $\mathrm{e}$, antes de seu abate, ele foi igualmente medido (mesmas grandezas mencionadas anteriormente). Após o abate, todas as suas folhas foram retiradas e pesadas, sendo que uma amostra de 100 folhas coletadas aleatoriamente ao longo da copa foi pesada separadamente. Uma vez em ambiente de laboratório, as 100 folhas da amostra tiveram as áreas de seus limbos medidas por meio do aparelho de planimetria óptica (LI-3 100) e foram levadas para a estufa a $60^{\circ} \mathrm{C}$ até que o peso estabilizasse. Com os valores do peso seco da amostra e de sua área foliar, foi possível determinar a Área Foliar Específica (AFE) do indivíduo, a qual relaciona a quantidade de área de folha por massa seca $\left(\mathrm{m}^{2} \cdot \mathrm{Kg}^{-1}\right)$. A partir da razão massa seca/ massa úmida da amostra, foi possível estimar o peso seco total das folhas da árvore abatida e, multiplicando-a pela AFE, estimou-se a área foliar total da árvore abatida.

O segundo trabalho de campo foi realizado entre 19 e 28 de setembro de $201 \mathrm{I}$, quando as mesmas 36 parcelas foram revisitadas e os mesmos procedimentos já descritos foram efetuados.

\section{Cálculo do IAF a partir da amostragem destrutiva}

Jonckheere et al. (2004) classificam a maneira como foi determinado o IAF neste trabalho como método da "árvore modelo" e, segundo Bréda (2003), é menos destrutivo, já que, a partir da amostragem de poucos indivíduos representativos do povoamento, pode-se extrapolar a área foliar, por meio de suas relações com variáveis dendrométricas das árvores. $\mathrm{Na}$ tentativa de definir equações alométricas que predissessem a área foliar das demais árvores, foram testadas diferentes combinações das medidas adquiridas dessa variável com a $\mathrm{H}$, o Rc e o DAP. No entanto, em razão da diversidade de clones e idades avaliada, não foram detectadas relações estatísticas significativas, principalmente para os dados coletados na segunda oportunidade. Como a relação da área foliar com o DAP foi a melhor, inclusive em outros trabalhos, como o de Dovey e Du Toit (2006) e Le Maire et al. (20II), a área foliar de cada uma das árvores da parcela foi estimada com base na relação entre o DAP e a área total de folhas do exemplar abatido. Dessa maneira, o cálculo do IAF se deu pela razão entre a soma da área foliar de todas as árvores e a área da parcela $\left(400 \mathrm{~m}^{2}\right)$.

\section{Obtenção do IAV a partir de fotografias hemisféricas}

Foi utilizada uma máquina fotográfica digital Nikon dotada de uma lente olho-de-peixe SIGMA EX DG $8.0 \mathrm{~mm}$, um tripé, nível de bolha e uma bússola para a tomada de fotografias verticais hemisféricas, orientadas ao norte magnético, no interior das parcelas. Em cada parcela foram tomadas 6 (seis) fotografias, procurando descrever uma trajetória triangular em torno do ponto central da parcela. Nos vértices desse triângulo imaginário, bem como em pontos intermediários dos seus lados, eram tomadas então as fotografias.

As fotografias foram tomadas em horários do dia nos quais não havia penetração de radiação solar direta no interior dos dosséis, preferencialmente um pouco antes do nascer do Sol e logo após o poente. Além disso, preocupou-se com as condições atmosféricas, já que a presença de nuvens confunde a classificação dos elementos da imagem.

As fotografias resultantes foram submetidas ao processamento no aplicativo HemiView 2.I, sendo que as devidas configurações foram definidas, como as coordenadas do centro de cada uma das parcelas, a declinação magnética e a altitude média. Um mesmo centro de circunferência e raio de análise foram fixados para o processamento de todas as fotografias, sendo que foi determinado um limiar (threshold) de classificação por parcela.

\section{Obtenção do IAV através do LAI-2000}

Concomitantemente à tomada de fotografias hemisféricas, foram realizadas medidas no interior do dossel com o equipamento LICOR LAI-2000. O aparelho era dotado de dois dispositivos sensitivos: um foi mantido fora dos plantios e em condições de iluminação ambiente, tomando medidas de iluminação difusa a cada 30 segundos, enquanto o outro foi levado ao interior das parcelas, onde, simultaneamente, foram efetuadas 25 medidas em um caminhamento que intercalava linhas e entrelinhas de plantio. Após essa etapa de coleta de dados, dava-se prosseguimento ao processamento dos dados que resultava em valores de IAF médios para cada parcela. 


\section{Análise estatística}

Ao final das coletas de dados em campo e do processamento dos dados, resultaram valores de IAF e IAV para cada uma das 36 parcelas segundo as três metodologias descritas. A comparação entre as metodologias foi feita mediante a aplicação de testes de significância dos parâmetros de equações de regressão linear. A ideia que norteou essa aplicação foi originada no fato de que a comparação envolvia amostras pareadas, cujas médias em termos absolutos não poderiam ser comparadas entre si. Assim, mais importante do que avaliar diferenças significativas entre valores médios de IAF e IAV resultantes da aplicação das três metodologias em questão, seria mais interessante avaliar a aderência das estimativas em cada ponto e o comportamento dessas estimativas nas 36 parcelas.

Assumindo $\circ$ método destrutivo como referência, as comparações se seguiram entre ele e os dois demais nas duas ocasiões (dois trabalhos de campo). Logo, consideraram-se as hipóteses de $\beta_{0}=0$ e $\beta_{1}=I$, ou seja, que no estabelecimento da regressão linear entre os valores de dois métodos, se $\beta_{0}=0$ e $\beta_{1}=1$, os valores de IAF observados em cada parcela seriam exatamente os mesmos, pois a reta resultante passaria pela origem dos eixos $x$ e y e a inclinação dessa reta seria de $45^{\circ}$ (tangente de $45^{\circ}=1$ ).

Os testes estatísticos foram, então, aplicados para testar essas duas hipóteses. Em primeiro lugar, testou-se a significância da regressão, ou seja, se $\beta_{\text {। }}$ seria diferente de 0 . Em seguida, foram testadas as hipóteses $\beta_{0}=0$ e $\beta_{1}=I$, por meio da aplicação de um teste simultâneo a $5 \%$ de significância. $O$ resultado dessa aplicação definiu um intervalo de confiança para valores de $\beta_{1}$, assumindo $\beta_{0}=0$.

\section{RESULTADOS E DISCUSSÃO}

A partir dos dados coletados nos dois trabalhos de campo e da aplicação dos três métodos descritos anteriormente, foi possível calcular as médias dos valores de IAF e IAV para o campo I (final do período chuvoso) e campo 2 (período seco). Cabe ressaltar que foi localizada uma incoerência no registro do valor do peso úmido total da parcela I e, por conseguinte, foram incorporados somente 35 dos resultados de IAF obtidos pelo método destrutivo e de IAV obtidos pelos métodos indiretos a essa análise. Os resultados são apresentados na Tabela I.

Os valores de IAF foram superiores aos de IAV nas duas oportunidades de coleta de dados em campo. Observa-se, ainda, que a aplicação do LAl-2000 resultou
TABELA I Valores médios de IAF e IAV obtidos a partir da aplicação dos três métodos.

TABLE I Mean values of LAI and VAl obtained by the three methods.

\begin{tabular}{lcc}
\hline & Campo I & Campo 2 \\
\hline Destrutivo & 3,09 & 2,28 \\
Fotos & 2,10 & 1,65 \\
LAl - 2000 & 2,59 & 1,91 \\
\hline
\end{tabular}

em estimativas de médias de IAV ligeiramente maiores em relação ao método fundamentado no uso de fotografias hemisféricas. Pesquisadores como Dovey e Toit (2006), MacFarlane et al. (2007), Paiva (2009) e Thimonier et al. (20I0) também reportaram a subestimação do IAV quando estimado com o LAl-2000 ou com as fotografias hemisféricas. Essa tendência dos métodos indiretos em subestimar os valores de IAV em dosséis de porte florestal é discutida por Bréda (2003) e Jonckheere et al. (2004), que a atribuem principalmente ao fato de que o modelo utilizado por esses instrumentos para o cálculo do IAV assume que a distribuição espacial do dossel florestal é uniforme, de maneira que não levam em consideração fatores de aglomeração e sobreposição das folhas.

Os três métodos foram capazes de detectar a diminuição dos valores de IAF e de IAV referentes à segunda oportunidade de coleta de dados (período seco) em relação à primeira (final do período chuvoso), como ressaltado em trabalhos como os de Chaves et al. (2007), Monte et al. (2007) e Paiva (2009).

A seguir, são apresentados os gráficos referentes à comparação dos resultados obtidos pelo método destrutivo e ao empregar o LAI-2000 (Figura $3 a)$ e as fotografias hemisféricas (Figura 3b) para as duas oportunidades de coleta (campo I, referente ao período chuvoso, e campo 2 , referente ao período seco). Os valores indicados com sombreamento foram superiores a $5 \%$ de significância, enquanto aqueles evidenciados em negrito, o contrário. Optou-se por incluir comparações entre os métodos envolvendo o emprego das fotografias hemisféricas e do LAI-2000 com o objetivo de avaliar a similaridade ou diferença das estimativas de IAV entre eles (Figura 3c).

Costuma-se avaliar comparações entre variáveis concentrando atenção nos valores dos coeficientes de correlação ou até mesmo nos valores de $R^{2}$. No entanto, se optou por discuti-las com alguma profundidade. Ao observar os valores de $\mathrm{R}^{2}$ apresentados na Figura 2 , constata-se que os valores mais elevados foram encontrados para os dados referentes ao primeiro campo (final do período chuvoso), quando os dosséis analisados apresentaram os maiores valores de IAF e 
(a)

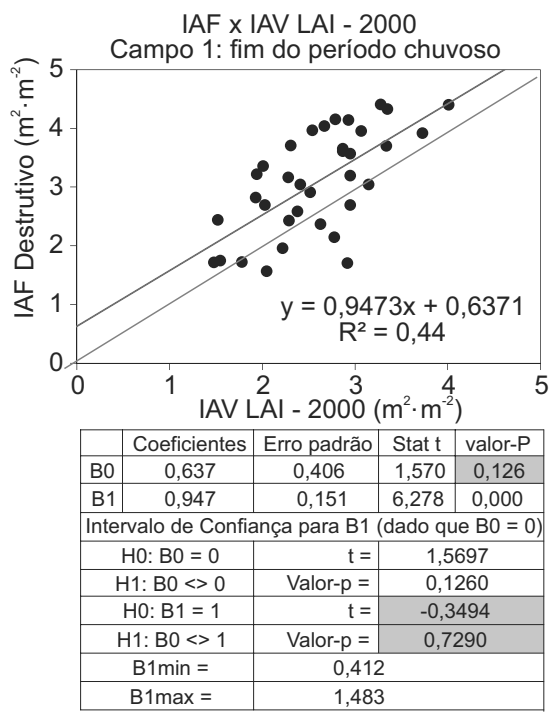

IAF $x$ IAV Fotografias

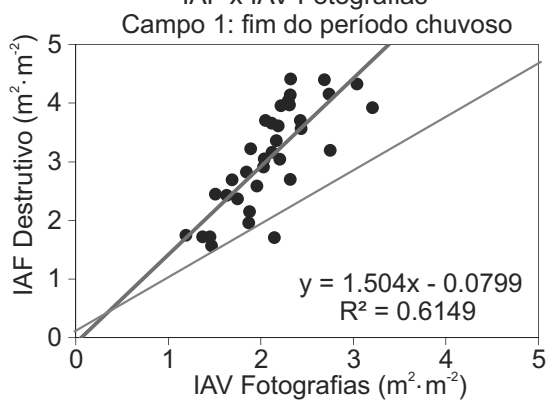

\begin{tabular}{|c|c|c|c|}
\hline & Coeficientes & Erro padrão & Stat t \\
\hline
\end{tabular}

\begin{tabular}{|c|c|c|c|c|}
\hline B0 & $-0,080$ & 0,354 & $-0,225$ & 0,823 \\
\hline B1 & 1,504 & 0,162 & 9,258 & 0,000 \\
\hline \multicolumn{2}{|c|}{ Intervalo de Confiança para B1 (dado que B0 =0) } \\
\hline H0: B0 $=0$ & $\mathrm{t}=$ & $-0,2253$ \\
\hline H1: $\mathrm{B} 0<>0$ & Valor- $\mathrm{p}=$ & 0,8231 \\
\hline $\mathrm{H} 0: \mathrm{B} 1=1$ & $\mathrm{t}=$ & 3,1026 \\
\hline H1: $\mathrm{B} 0<>1$ & Valor- $\mathrm{p}=$ & $\mathbf{0 , 0 0 3 9}$ \\
\hline
\end{tabular}

AV Fotografias x IAV LAI - 2000 Campo 1: fim do período chuvoso

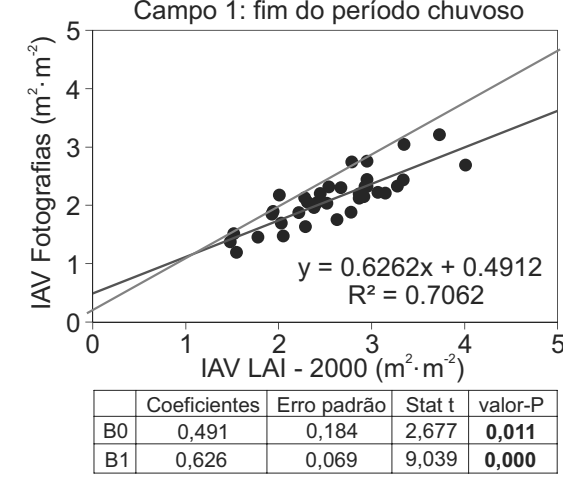

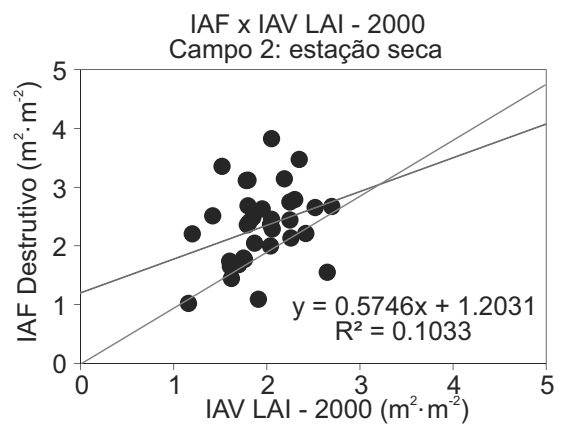

\begin{tabular}{|c|c|c|c|c|}
\hline & Coeficientes & Erro padrão & Stat t & valor-P \\
\hline B0 & 1,203 & 0,569 & 2,115 & $\mathbf{0 , 0 4 2}$ \\
\hline B1 & 0,575 & 0,290 & 1,979 & $\mathbf{0 , 0 5 6}$ \\
\hline
\end{tabular}

IAF x IAV Fotografias

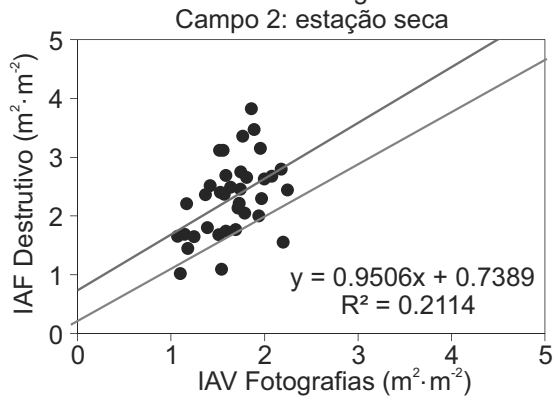

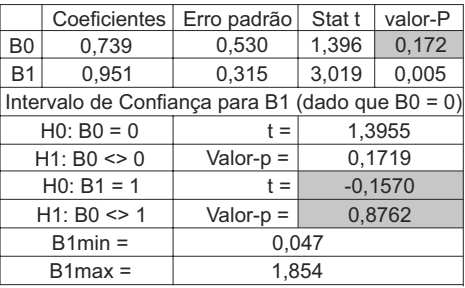

Fotografias x LAl - 2000 Campo 2: estação seca

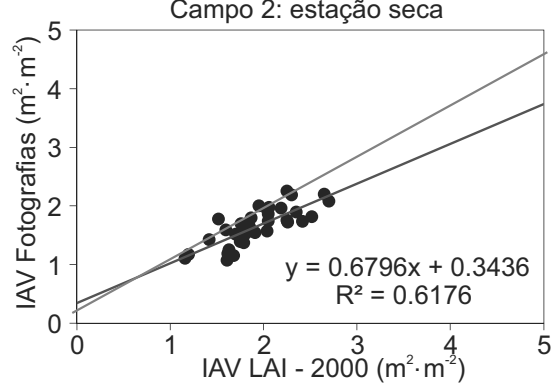

\begin{tabular}{|c|c|c|c|c|}
\hline & Coeficientes & Erro padrão & Stat $\mathrm{t}$ & valor- $\mathrm{P}$ \\
\hline B0 & 0,344 & 0,180 & 1,912 & 0,064 \\
\hline B1 & 0,680 & 0,092 & 7,411 & 0,000 \\
\hline Intervalo de Confiança para B1 & dado que $\mathrm{B} 0=0$ ) \\
\hline \multicolumn{2}{|c|}{ H0: $\mathrm{B} 0=0$} & $\mathrm{t}=$ & 1,9122 \\
\hline H1: $\mathrm{B} 0<>0$ & Valor- $\mathrm{p}=$ & 0,0643 \\
\hline $\mathrm{H} 0: \mathrm{B} 1=1$ & $\mathrm{t}=$ & $-3,4947$ \\
\hline H1: $\mathrm{B} 0<>1$ & Valor- $\mathrm{p}=$ & $\mathbf{0 , 0 0 1 3}$ \\
\hline
\end{tabular}

FIGURA 3 Resultados da aplicação dos testes estatísticos para as comparações entre os métodos de estimativa de IAF e IAV em duas oportunidades de coleta de dados em campo (período chuvoso e período seco).

FIGURE 3 Statistical tests results for comparisons between methods of estimating LAl and VAl in two opportunities for field data collection (rainy season and dry season). 
de IAV. Ainda para este período, as relações entre IAF e IAV (método destrutivo $x$ fotografias hemisféricas ou LAI-2000) apresentaram valores significativos para $\beta_{0}=0$ e $\beta_{1}=I$, indicando que, apesar de relativamente fracas relações, as variáveis apresentam algum grau de aderência a $5 \%$ de significância. Vale salientar que, nesses casos, estaríamos considerando grande dispersão dos pontos em torno da reta de regressão $\circ$ que implicaria que $\beta_{0}=0$ e $\beta_{1}=$ I seriam resultados possíveis apenas dentro de uma série de eventos. Para a relação entre IAV estimado pelas fotografias hemisféricas e pelo uso do LAI-2000, verificou-se valor mais elevado de $R^{2}$, mas $\beta_{0}$ não assumiria valor 0 e nem $\beta_{\text {, assumiria o valor }}$ I a $5 \%$ de significância, indicando que apesar de mais fortemente relacionadas, não seria obtida uma relação direta entre as variáveis.

Ao observar os resultados alcançados para o segundo campo (período seco), as relações entre IAF e $I A V$, este último estimado pelos dois métodos indiretos, os valores de $R^{2}$ foram sempre muito baixos e apesar dos resultados $\beta_{0}=0$ e $\beta_{1}=1$ serem possíveis a $5 \%$ de significância, a aderência entre as variáveis foi muito fraca. Para a relação de IAV estimado pelos dois métodos indiretos, valem os mesmos comentários apresentados para os dados do período chuvoso (campo I), ou seja, relação mais consistente, porém não seria encontrada relação direta com $\beta_{0}=0$ e $\beta_{1}=$ I a $5 \%$ de significância entre as estimativas dos dois métodos. Thimonier et al. (2010) também se depararam com resultados semelhantes.

Essa diferença dos resultados alcançados nas duas oportunidades de coleta de dados pode ser explicada pelo fato de que em condições de menor densidade foliar e, consequentemente, sob dosséis descontínuos, o potencial dos métodos fundamentados na transferência radiativa diminui (JONCKHEERE et al., 2004). Essas limitações no cálculo do balanço de energia que efetivamente chega ao interior do dossel em relação à quantidade de energia que chegou ao seu topo foi também abordado por Bréda (2003) e MacFarlane et al. (2007).

\section{CONCLUSÕES}

Em termos absolutos as estimativas de IAF e de IAV diferem entre si, com tendência de o método destrutivo resultar em valores superiores àqueles alcançados a partir de métodos fundamentados na teoria de transferência radiativa. Os três métodos foram capazes de detectar diminuição nos valores de IAF e de IAV com a mudança da estação do ano, sendo que a utilização das fotografias hemisféricas ou do LAI-2000 mostrou-se indiferente nas duas estações do ano. Com a diminuição da densidade de folhas no dossel, assumindo o método destrutivo como referência, o emprego das fotografias hemisféricas ou do LAI-2000 resultaria em estimativas significativamente diferentes.

\section{AGRADECIMENTOS}

Gostaríamos de externar nossos mais sinceros agradecimentos aos profissionais da FIBRIA S/A das unidades Jacareí (SP), Aracruz (ES) e São Mateus (ES) pelo imprescindível apoio à coleta de dados em campo e seu processamento. Agradecimentos estendidos ao Conselho Nacional de Desenvolvimento Científico e Tecnológico (CNPq) pela concessão de bolsa de Mestrado da Engenheira Florestal Roberta Zecchini Cantinho.

\section{REFERÊNCIAS}

BRÉDA, N. J. J. Ground-based measurements of leaf area index: a review of methods, instruments and current controversies. Journal of Experimental Botany, Oxford, v. 54, n. 392, p. 2043-24I7, Nov. 2003.

CAMPBELL, G. S.; NORMAN, J. M. The description and measurement of plant canopy structure. In: RUSSELL, G.; MARSHALL, B.; JARVIS, P. (Ed.). Plant canopies: their growth, form and function. Cambridge: Cambridge University, 1989. p. I-19.

CHAVES, R. A.; REIS, M. G. F; REIS, G. G.; PEZZOPANE, J. E. M.; XAVIER, A.; MONTE, M. A. Dinâmica de cobertura de dossel de povoamentos de clone de Eucalyptus grandis W. Hill ex-Maiden submetidos a desrama artificial e desbaste. Revista Árvore, Viçosa, v. 31, n. 6, p. 989-998, 2007.

DOVEY, S. B.; TOIT, B. Calibration of LAI-2000 canopy analyzer with leaf area index in a young eucalypt stand. Trees, Berlin, v. 20, p. 273-277, 2006.

EMPRESA BRASILEIRA DE PESQUISA AGROPECUÁRIA. Centro Nacional de Pesquisa de Solos. Sistema brasileiro de classificação de solos. 2. ed. Rio de Janeiro, 2006. 306 p.

GOWER, S. T.; KUCHARIK, C. J.; NORMAN, J. M. Direct and indirect estimation of leaf area index, $f_{A P A R}$, and net primary production of terrestrial ecosystems. Remote Sensing of Environment, New York, v. 70, p. 29-5I, 1999.

JONCKHEERE, I.; FLECK, S.; NACKAERTS, K.; MUYSA, B.; COPPIN, P.; WEISS, M.; BARET, F. Review of methods for in situ leaf area index determination: part I, theories, sensors and hemispherical photography. Agricultural and Forest Meteorology, Amsterdam, v. I21, p. 19-35, 2004. 
LANG, A. R. G.; MCMURTRIE, R. E. Total leaf areas of single trees of Eucalyptus grandis estimated from transmittances of the sun's beam. Agricultural and Forest Meteorology, Amsterdam, v. 58, p. 79-92, 1992.

LARCHER, W. Ecofisiologia vegetal. São Carlos: Rima, 2006. $550 \mathrm{p}$.

LE MAIRE, G.; MARSDEN, C.; VERHOEF, W.; PONZONI, F. J.; LO SEEN, D.; BÉGUÉ, A.; STAPE, J.; NOUVELLON, $Y$. Leaf area index estimation with MODIS reflectance time series and model inversion during full rotations of Eucalyptus plantations. Remote Sensing of Environment, New York, v. II5, n. 2, p. 586-599, $201 \mathrm{I}$.

LOWMAN, M. D.; NADKARNI, N. M. Forest canopies. San Diego: Academic, 1995. 624 p.

MACFARLANE, C.; ARNDT, S. K.; LIVESLEY, S. J.; EDGAR, D. A. C. A.; ADAMS, M. A.; EAMUS, D. Estimation of leaf area index in eucalypt forest with vertical foliage, using cover and fullframe fisheye photography. Forest Ecology and Management, Amsterdam, v. 242, p. 756-763, 2007.

MAESTRI, R. Modelo de crescimento e produção para povoamentos clonais de Eucalyptus grandis considerando variáveis ambientais. 2003. I43 p. Tese (Doutorado em Ciências Florestais) - Universidade Federal do Paraná, Curitiba, 2003.

MONTE, M. A.; REIS, M. G. F.; REIS, G. G.; LEITE, H. G.; STOCKS, J. J. Métodos indiretos de estimação da cobertura de dossel em povoamentos de clone de eucalipto. Pesquisa Agropecuária Brasileira, Brasília, v. 42, n. 6, p. 769-775, jun. 2007.

O'BRIEN, M. J. P. Ecologia e modelamento de florestas tropicais. Belém: FCAP, 1995. 400 p.

OMETTO, J. C. Bioclimatologia vegetal. São Paulo: Ceres, 1981. 425 p.
PAIVA, Y. G. Estimativa do Índice de Área Foliar por métodos óticos e sensoriamento remoto para calibrar modelo ecofisiológico em plantios de eucalipto em áreas de relevo ondulado. 2009. 62 p. Dissertação (Mestrado em Metereologia Agrícola) - Universidade Federal de Viçosa, Viçosa, 2009.

RODRÍGUEZ, R.; REAL, P.; ESPINOSA, M.; PERRY, D. A. A process-based model to evaluate site quality for Eucalyptus nitens in the Bio-Bio Region of Chile. Forestry, Oxford, v. 82, n. 2, p. 149-162, 2009.

ROSS, J. The radiation regime and architecture of plant stands. London: Junk, 1981. 39I p.

SOUZA, M. J. H.; RIBEIRO, A.; LEITE, H. G.; LEITE, F. P.; MINUZZI, R. B. Disponibilidade hídrica do solo e produtividade do eucalipto em três regiões da bacia do Rio Doce. Revista Árvore, Viçosa, v. 30, n. 3, p. 399-4I 0, 2006.

STAPE, J. L. Eucalyptus production and supply, use and efficiency of the use of water, light and nitrogen across a geographic gradient in Brazil. Forest Ecology and Management, Amsterdam, v. 193, p. 17-31, 2004.

STAPE, J. L.; RYAN, M. G.; BINKLEY, D. Testing the utility of the 3-PG model for growth of Eucalyptus grandisurophylla with natural and manipulated supplies of water and nutrients. Forest Ecology and Management, Amsterdam, v. 193, p. 219-234, 2004.

THIMONIER, A.; SEDIVY, I.; SCHLEPPI, P. Estimating leaf area index in different types of mature forest stands in Switzerland: a comparison of methods. European Journal of Forest Research, Georgetown, v. I29, p. 543-562, 2010.

XAVIER, A. C.; VETTORAZZI, C. A. Índice de área foliar de coberturas em uma microbacia hidrográfica subtropical. Scientia Agrícola, Piracicaba, v. 60, n. 3, p. 425-43I, 2003.

ZHANG, Y.; CHEN, J. M.; MILLER, J. R. Determining digital hemispherical photograph exposure for leaf area index estimation. Agricultural and Forest Meteorology, Amsterdam, v. I33, p. I66-I8I, 2005. 